This material is presented to ensure timely dissemination of scholarly and technical work. Copyright and all rights therein are retained by authors or by other copyright holders. All persons copying this information are expected to adhere to the terms and constraints invoked by each author's copyright. In most cases, these works may not be reposted without the explicit permission of the copyright holder. (C2006 IEEE. Personal use of this material is permitted. However, permission to reprint/republish this material for advertising or promotional purposes or for creating new collective works for resale or redistribution to servers or lists, or to reuse any copyrighted component of this work in other works must be obtained from the IEEE."

\title{
Detectors for the 10000 Pixel SCUBA-2 Super- conducting Sub-mm Camera for Astronomy
}

\author{
Adam L. Woodcraft (for the SCUBA-2 consortium) \\ SUPA, Institute for Astronomy, University of Edinburgh, Royal Observatory, Blackford Hill, Edinburgh, EH9 3HJ, UK \\ adam.woodcraft@physics.org
}

\begin{abstract}
We describe the current status of the SCUBA-2 sub-mm camera for astronomy.
\end{abstract}

Current continuum (broadband) instruments for submillimetre astronomy (wavelengths of a few hundred $\mu \mathrm{m}$ to a few $\mathrm{mm}$ ) are severely limited by the small number of pixels. Instruments have moved from a single pixel to hundreds over the last decade, and further increases in pixel count are necessary to exploit existing and future telescopes. Another aim is to achieve background limited performance (BLIP), in which the noise limit is set by photon noise from background radiation. Bolometric detection is used, since this is the most sensitive method. Until recently instruments have generally used germanium semiconducting thermometers. However, despite having reached the fundamental noise limits, these detectors are not background limited for the best ground based telescopes. It is also difficult to make arrays because the germanium chips must be individually glued to each pixel, and because they cannot be multiplexed.

Superconducting detectors (transition edge sensors) are therefore being used for the next generation of instruments. As well as being able to achieve better signal to noise than semiconducting bolometers, it is possible to fabricate an entire array without any operations being required at the level of a single pixel. Moreover, a multiplexed readout can be used.

However, the amount of wiring required between a detector array and multiplexer chip has set a practical limit on the array size. For SCUBA-2, array sizes of over 1000 pixels (Fig. 1) are achieved by using a new multiplexer configuration, in which the multiplexer wafer is bonded to the detector wafer, with indium bump bonds providing electrical connections between each pixel and the multiplexer. These arrays can be butted together on two sides, to form focal planes of over 5000 pixels. A time division multiplexing scheme (TDM) is used, with multiplexers supplied by NIST. The detectors themselves consist of $\mathrm{Mo} / \mathrm{Cu}$ bi-layer thermistors, with weak thermal linking provided by a silicon nitride membrane

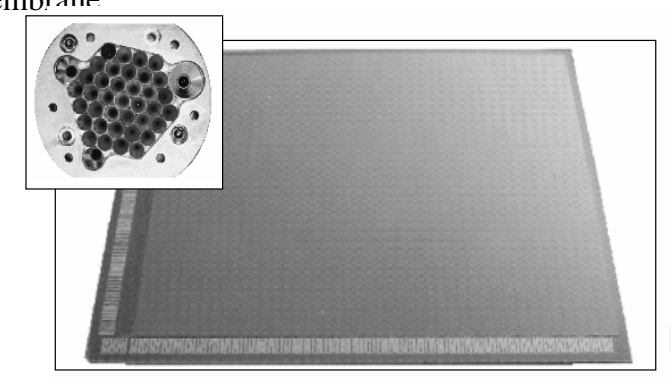

Figure 1: A 32x40 SCUBA-2 array; the inset shows a previous generation 40 pixel array (SCUBA-1)

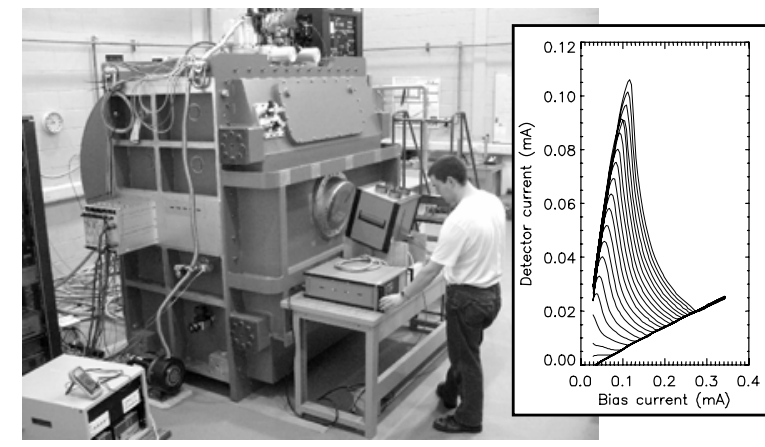

Figure 2: The SCUBA-2 instrument. The inset shows instrument characterisation measurements [1] on one pixel

To obtain the required performance, the arrays must be operated from a heat sink at a temperature of approximately $0.06 \mathrm{~K}$, with cooling to this temperature provided by a dilution refrigerator. This operates from a temperature of $4 \mathrm{~K}$, which traditionally would be provided by a liquid helium bath. To avoid the running costs associated with liquid helium consumption, the instrument is instead mechanically cooled using pulse tube coolers.

The size of the instrument (over $5 \mathrm{~m}^{3}$; Fig. 2) is driven by the large mirrors required due to the large field of view. The last three mirrors must be cooled to below $10 \mathrm{~K}$ in order to reduce the thermal background on the arrays.

SCUBA-2 will operate simultaneously at two wavelength ranges centred on 450 and 850 microns (670 and $350 \mathrm{GHz}$ ). So far, a prototype array designed for each wavelength range has been tested in a dedicated testbed, with results meeting the specifications. One array has also been tested in the instrument itself, demonstrating that the instrument performs well. Optical noise equivalent powers (NEP) of below $1.4 \times 10^{-16} \mathrm{~W} \mathrm{~Hz}^{-0.5}$ and $2.5 \times 10^{-17} \mathrm{~W} \mathrm{~Hz}^{-0.5}$ for the 450 and $850 \mu \mathrm{m}$ arrays respectively have been measured; both values are within the specifications. The success of tests to date has enabled the production of science grade arrays to start, with delivery of the instrument to the telescope (JCMT, Mauna Kea, Hawaii) expected in late 2006. Further information on the instrument and detectors, and the many groups involved in the design and construction, can be found in [1] $\&[2]$.

\section{REFERENCES}

[1] Adam L. Woodcraft, Matthew I. Hollister, Dan Bintley, Maureen A. Ellis, Xiaofeng Gao, Wayne S. Holland et. al, "Characterization of a prototype SCUBA-2 1280 pixel submillimetre superconducting bolometer array," Proc. SPIE, vol. 6275, pp. 62751F-1-11, 2006.

[2] Wayne Holland, Michael MacIntosh, Alasdair Fairley, Dennis Kelly, David Montgomery, David Gostick et. al, "SCUBA-2: a 10,000 pixel submillimeter camera for the James Clerk Maxwell Telescope," Proc. SPIE, vol. 6275, pp. 62751E-1-15, 2006. 